\title{
Uso de harina de hojas de quínoa (Chenopodium quinoa) como ingrediente innovador, para la elaboración de alimentos de uso humano
}

\author{
Use of quinoa (Chenopodium quinoa) leaves' flour as an innovative ingredient in \\ human food elaboration
}

Luis Sáez-Tonacca ${ }^{1 *}$, Alejandra Aravena-Narbona ${ }^{1}$, Carlos Díaz Ramírez.

\begin{abstract}
RESUMEN
La quínoa (Chenopodium quinoa) es un pseudocereal cultivado ampliamente en países andinos, con alta variabilidad genética, adaptabilidad y bajo costo de producción, razón por la cual sus granos constituyen un alimento de gran importancia en países como Bolivia y Ecuador, donde además las hojas del quínoa se emplean también como hortalizas. En este contexto, surge la idea de deshidratar hojas de quínoa para elaborar harina de hojas, como una alternativa de nuevo producto en la elaboración de alimentos para consumo humano. En primera instancia se midió el rendimiento de hojas verdes recolectadas en un predio ubicado en la región del Libertador Bernardo O’Higgins, posteriormente, las hojas recolectadas fueron deshidratadas y molidas a fin de elaborar harina de hojas de quínoa. En la segunda etapa del estudio, se empleó la harina de hojas en la elaboración de galletas de avena con niveles decrecientes de incorporación al 20, 15 y $10 \%$ de harina de hojas respectivamente.

Los resultados sugieren que existen diferencias en la aceptabilidad en función del nivel de incorporación de harina de hojas. Adicionalmente se evaluó la disposición por parte de los participantes a adquirir otros productos que incluyeran harina de hojas de quínoa.
\end{abstract}

Palabras clave: quínoa, consumidor, aceptación, nivel de agrado, harina de hojas de quínoa.

\begin{abstract}
Quinoa (Chenopodium quinoa) is a widely cultivated pseudo-cereal in Andean countries, with high genetic variability, adaptability and low production cost, reason why their grains are a highly important source of food in countries such as Bolivia and Equator, where quinoa leaves are also used as vegetables. In this context, the idea of dehydrating quinoa leaves arises as new ingredient alternative for human consumption. On the first stage of study, yield on green quinoa leaves cultivated at the Bernardo $O^{\prime} H i g g i n s$ region was measured, later; collected leaves were dehydrated and grounded in order to make quinoa leaves flour. On the second stage, quinoa leaves flour was used to prepare oat biscuits with decreasing amounts of quinoa leaves flour at 20, 15 and $10 \%$ respectively.

The results indicate that there are differences in acceptability depending on the level of incorporation of leaf meal. Additionally, the willingness of the participants to purchase other products that included quinoa leaf meal was evaluated.
\end{abstract}

Key words: quinoa, consumer, acceptance, pleasantness, flour made of quinoa leaves.

\section{Introducción}

En cultivo de la quínoa (Chenopodium quinoa Willd.), se caracteriza por su alta calidad nutricional, siendo una fuente importante de proteínas (FAO, 2011), no obstante, posee saponinas que dificultan la absorción de algunos nutrientes y son por lo general, removidas del episperma del grano mediante el lavado con agua, aumentando con ello el costo de producción.

En el caso de Chile, el cultivo corresponde principalmente a la agricultura familiar, con superficies reducidas (Bazile et al., 2014), cuya superficie de acuerdo al Censo agropecuario de 2007, alcanzó las 1.468 hectáreas, con una producción total de 883 toneladas y un rendimiento promedio nacional

1 Universidad de Santiago, Facultad Tecnológica, Departamento Gestión Agraria. Santiago, Chile.

* $\quad$ Autor para correspondencia: luis.saez@usach.cl

Fecha de Recepción: 11 marzo, 2017.

Fecha de Aceptación: 19 mayo, 2018.

DOI: http://dx.doi.org/10.4067/S0718-34292018005000602: Publicado en línea: 24-agosto-2018. 
de 0,6 ton/ha, siendo Tarapacá la principal región productora, con el $95 \%$ de la producción nacional (Muñoz, 2013). En 2012 la superficie total de cultivo se estimó en 1.779 ha (INE, 2016).

En relación a los aspectos de manejo, es conocida la necesidad de incorporar prácticas de producción y post-cosecha que contribuyan a mejorar la competitividad de la producción en Chile, (Delatorre-Herrera et al., 2013), como por ejemplo en la desaponificación del grano. En este sentido, se observa en países como Bolivia, Perú y Ecuador el uso de hojas frescas de quínoa en la alimentación humana, como hortalizas en reemplazo de acelga y la espinaca, o bien como forraje (FAO, 2011, Meyhuay, 2013) y estudios previos sugieren que por cada kilo de grano de quínoa producido, es posible cosechar hasta dos kilos y medio de hojas frescas, aunque estas por lo general son desechadas en el campo (ANDINA, 2013). Otros estudios indican que desde el punto de vista nutricional y de rendimiento, la época oportuna para la recolección de hojas de quínoa para la alimentación humana es poco antes del inicio de la floración, entre los 60 a 80 días después de la germinación (Mujica et al., 1999), denominándose a estas hojas se les denomina como "hojas tiernas".

Debido a lo anterior, y ante la ausencia de información referente al uso de hojas de quínoa en Chile, la presente trabajo se propuso la presente investigación con la finalidad de generar información sobre la producción de hojas frescas de quínoa y evaluar la aceptabilidad por parte de consumidores hacia alimentos que incluyeran harina de hojas de quínoa como ingrediente.

\section{Materiales y Métodos}

\section{Obtención y transformación de la materia prima}

La extracción de la materia prima base para la investigación se realizó en la comuna de Paredones, Región del Libertador Bernardo O'Higgins, entre los días 26, 27 y 28 de enero del año 2016, en un cultivo que se encontraba alrededor de los 60-70 días de estado fenológico.

Las hojas "tiernas" fueron extraídas desde un sector del cultivo, correspondiente a 3 hileras distribuidas en una superficie de 4,7 $\mathrm{m}$ de ancho por $96 \mathrm{~m}$ de largo $\left(451 \mathrm{~m}^{2}\right)$, recolectándose un total de 5.622 hojas, desde 350 plantas. Se escogieron las hojas más verdes, tiernas y sanas, excluyendo aquellas con tonos amarillos, recolectando de 4 a 10 hojas de aquellas plantas de hasta 1,40 m y más de 35 hojas en las plantas de hasta $2,10 \mathrm{~m}$. El total de hojas tiernas recolectadas fue de $11.500 \mathrm{~g}$.

Se realizó una segunda selección con el fin descartar aquellas hojas con tonos amarillos o café, así como manchas que no hubiesen sido detectadas durante la recolección, adicionalmente las hojas se lavaron en una solución se agua potable con hipoclorito de sodio.

Las hojas fueron deshidratadas de forma natural con luz solar desde el 30 de enero al 2 de febrero a una temperatura ambiente de $32^{\circ} \mathrm{C}$, aproximadamente. Una vez deshidratadas, las hojas fueron trituradas manualmente y posteriormente molidas mediante un molinillo manual. El rendimiento obtenido fue de $1.700 \mathrm{~g}$ de harina de hojas de quínoa deshidratadas, de color verde, aroma y textura a hierba y aspecto de polvo fino. Fueron empacadas en bolsas herméticas de polipropileno, en formato de 350 a $300 \mathrm{~g}$ hasta la formulación del producto experimental.

La harina de hojas de quínoa se sometió a un análisis químico proximal en el laboratorio del Departamento de Ciencia y Tecnología de los Alimentos de la Universidad de Santiago de Chile, bajo las normas chilenas aprobadas, para determinar su contenido de proteínas, cenizas y extracto no nitrogenado.

\section{Formulación y preparación del producto experimental}

Con la harina de hojas de quínoa se formuló una colación saludable, definida por el SERNAC (2003), como una preparación que se consume entre comidas habituales y que incorporan alimentos naturales o elaborados tales como lácteos, frutas frescas, frutos secos, verduras y cereales, con bajo aporte calórico. La galleta debería, idealmente, incorporar más de un $10 \%$ de la dosis diaria recomendada (DDR) de proteínas para un adulto.

Dada la ausencia de otros diseños experimentales, se realizaron pruebas hasta llegar a una receta palatable y atractiva de galletas de avena con harina de hojas de quínoa. Se tomó una receta base para 25 galletas saludables de avena compuesta por avena (115 g), harina integral de trigo (30 g), puré de manzana (40 g), azúcar rubia (50 g), aceite vegetal $(2 \mathrm{~mL})$, esencia de vainilla ( $1 / 4$ cucharadita) y polvos de hornear ( $1 / 4$ cucharadita) y se agregó 
diferentes porcentajes de harina de hojas de quínoa, disminuyendo la avena y manteniendo constante la harina de trigo, ya que esta es la que otorga consistencia a la receta. La formulación base no incluyó productos de origen animal. A esta receta se adjuntó porcentajes de 20,15 y $10 \%$ de harina de hojas de quínoa deshidratada y se incluyó un tratamiento paralelo lavando la harina de hojas deshidratadas con agua tibia e incluyéndolas en los mismos porcentajes en la receta (Tabla 1).

Tabla 1. Formulaciones para 25 galletas de 10 gramos.

\begin{tabular}{lcccc}
\hline Ingrediente & $\begin{array}{c}\text { Formulación } \\
(20 \%)\end{array}$ & $\begin{array}{c}\text { Formulación } \\
(15 \%)\end{array}$ & $\begin{array}{c}\text { Formulación } \\
(10 \%)\end{array}$ & Testigo \\
\hline $\begin{array}{l}\text { Harina de hojas } \\
\text { de quínoa, g }\end{array}$ & 33 & 25 & 17 & 0 \\
Avena, g & 82 & 90 & 98 & 115 \\
\hline
\end{tabular}

La masa fue preparada mezclando el puré de manzana con el azúcar rubia y la esencia de vainilla y revolviendo los ingredientes hasta su total homogeneización. Luego fue agregada y mezclada la harina de hojas de quínoa y dejada reposar durante 15 min, de forma que el azúcar se mezclase con las hojas y pudiese disminuir en parte el amargor que otorgan a la receta. Pasados los $15 \mathrm{~min}$, fueron agregados los demás ingredientes: avena, harina integral y polvos de hornear. Las galletas se cortaron con un diámetro de aproximadamente $3 \mathrm{~cm}$ y $10 \mathrm{~g}$ de peso y fueron horneadas durante $20 \mathrm{~min}$ a $150^{\circ} \mathrm{C}$.

Se incluyó a la evaluación la formulación base de la galleta de avena sin presencia de harina de hojas de quínoa (testigo o patrón), para poder comparar su aceptación respecto a los tratamientos adicionados con harina de hojas de quínoa. Al incluir 3 porcentajes de inclusión con ambos tratamientos, más una galleta testigo, la evaluación sensorial constó de 7 tratamientos distintos (Tabla 2).

Tabla 2. Tratamientos utilizados en la investigación.

\begin{tabular}{lc}
\hline Tratamiento & \% Inclusión y tratamiento utilizado \\
\hline 1 & $20 \%$ hojas sin lavar \\
2 & $15 \%$ hojas sin lavar \\
3 & $10 \%$ hojas sin lavar \\
5 & $20 \%$ hojas lavadas \\
6 & $15 \%$ hojas lavadas \\
7 & $10 \%$ hojas lavadas \\
0 & Testigo \\
\hline
\end{tabular}

Mediante cálculos teóricos se determinó el contenido de proteínas presente en las galletas elaboradas para la prueba, según los diferentes tratamientos aplicados.

\section{Evaluación sensorial}

La evaluación sensorial constó de la realización de pruebas hedónicas por atributos, una prueba de aceptación por ordenación y una encuesta cualitativa de aceptación. Estas se realizaron en dependencias de la Universidad de Santiago en 3 horarios distintos: 11:00 am, 15:00 pm y 17:00 pm durante marzo de 2016.

Se construyó un panel sensorial con 100 jueces afectivos, individuos no seleccionados ni adiestrados. El panel estuvo compuesto por personas entre $18 \mathrm{y}$ 57 años. Un $60 \%$ fue del sexo femenino y un $40 \%$ del sexo masculino. El $92 \%$ declaró ser soltero y un $8 \%$ casado. En relación a su ocupación, un $80 \%$ fueron estudiantes y un $20 \%$ trabajadores dependientes. Un 53\% del panel declaró participar ocasionalmente en las compras de alimentos de su hogar, mientras que un $15 \%$ declaró participar contantemente de estas. Un 32\% declaró no participar en estas compras. Respecto a su dieta, un $87 \%$ declaró alimentarse con una dieta tradicional, es decir, omnívora. Un 8\% declaró seguir una dieta vegetariana, un 3\% declaró poseer una dieta pescetariana $\mathrm{y}$, finalmente, un $2 \%$ declaró seguir una dieta vegana.

Para las pruebas hedónicas se utilizó una escala de 7 puntos para evaluar color, olor, textura, sabor y percepción general del producto (Hernández, 2005). Las muestras fueron entregadas al mismo tiempo y en órdenes distintos para evitar errores por posición y disminuir errores por contraste y fueron codificadas con un número aleatorio de 3 dígitos para evitar errores por expectación. También presentaron forma y envoltura similar para evitar errores por estimulación. Se entregaron a temperatura ambiente, en recipientes idénticos, que no comunicaron olor ni sabor alguno al alimento para no interferir con las percepciones de los panelistas (Fernández, 2005). Acompañando las muestras, se ofreció un vaso con agua para que el panelista utilizase como enjuague bucal entre muestras. Pudiendo, según la decisión del juez, ser ingerida o expulsada (Watts et al., 1992). Al disponer de las 7 muestras simultáneamente, los panelistas pudieron volver a evaluarlas si así lo deseasen y hacer comparaciones entre ellas (Ramírez-Navas, 2012) (Tabla 3).

Debido a que pruebas hedónicas solo estiman la intensidad de agrado por un producto, pero no proporcionan información con respecto al consumo, también se realizaron pruebas de aceptación por ordenamiento, con el objetivo de identificar 
Tabla 3. Escala hedónica para evaluación sensorial.

\begin{tabular}{lc}
\hline Categoría & Valor atributo \\
\hline Me gusta mucho & 7 \\
Me gusta moderadamente & 6 \\
Me gusta ligeramente & 5 \\
Ni me gusta ni me disgusta & 4 \\
Me disgusta ligeramente & 3 \\
Me disgusta moderadamente & 2 \\
Me disgusta mucho & 1 \\
Fuente: Watts et $a l .(1992)$. &
\end{tabular}

la muestra con más aceptación por parte del consumidor y compararla con la aceptación de la muestra testigo.

Para esto se pidió a los panelistas ordenar las muestras de acuerdo a su aceptación, evitando clasificar dos muestras en la misma posición, debiendo dar un valor diferente a cada muestra incluso si les pareciera similar, con valores del 1 hasta 7 , con 1 indicando mayor rechazo y el número 7 para la muestra con mayor aceptación.

Finalmente, se solicitó a los panelistas responder una encuesta a fin de obtener la percepción de estos como eventuales consumidores de otros productos enriquecidos o adicionados con harina de hojas de quínoa, y su disposición de compra. En esta etapa se les explicó el objetivo del estudio y la motivación de dar un uso a las hojas de quínoa. Esta encuesta se dejó para el final de la evaluación, para no interferir con la opinión de los panelistas durante la evaluación sensorial.

Los panelistas se dividieron en grupos de 15 a 30 personas. La evaluación sensorial se inició con la prueba hedónica por atributos, donde se les solicitó observar, oler, tocar, degustar y evaluar los 4 parámetros por separado y en conjunto, indicando su nivel de agrado en la escala hedónica de la tabla asignada. Entre las muestras se recordó a los panelistas enjuagar su boca con un poco de agua para eliminar el sabor residual de la muestra anterior. Se repitió el proceso en cada una de las 7 muestras.

A continuación, se realizó la prueba de aceptación por ordenación en 2 etapas. En la primera etapa se solicitó a los panelistas ordenar en un rango del 1 al 7 las muestras de acuerdo a los parámetros evaluados anteriormente. En la segunda etapa, mediante un cuestionario, se recopilaron percepciones importantes para la investigación hacia el concepto de enriquecer o adicionar alimentos con harina de hojas de quínoa.

\section{Análisis estadístico}

Los datos recopilados fueron tabulados en Microsoft Excel 2013 y sometidos a un análisis estadístico mediante el programa Statistical Package for the Social Sciences, versión 22.

El análisis de los datos obtenidos con la prueba hedónica por atributos se realizó mediante una comparación de medias y desviaciones estándar. Para determinar la existencia de diferencias significativas entre dichas medias, se utilizó la prueba no paramétrica de Kruskal Wallis. En aquellas que arrojó diferencias significativas, se aplicó la prueba no paramétrica de U de Mann-Whitney para comparar entre pares de tratamientos (Carpenter et al., 2003). La base teórica indica que el valor crítico de diferencia de suma de rangos, para un nivel de significancia del 5\%, con 7 tratamientos (incluido el control) y 100 panelistas es de 71.

El análisis estadístico para la prueba de aceptación por ordenamiento se realizó sumando el total de los valores de posición asignados a cada tratamiento y determinando las diferencias significativas entre los tratamientos con adición de hojas de quínoa deshidratadas y el tratamiento control, comparando los totales de los valores de posición de todos los posibles pares, utilizando la prueba de Friedman Newell (Pedrero y Pangborn, 1989).

\section{Resultados y Discusión}

\section{Disponibilidad de materia prima para recolección y rendimientos}

La densidad de plantas $/ \mathrm{m}^{2}$ (451 metros de área de cultivo/ total de plantas), se estima en 1.125 plantas de quínoa en el predio, con una densidad por hectárea de 8.888 plantas. El promedio de hojas posibles de extraer por planta (total de plantas/ total de hojas cosechadas durante la recolección), es de 16 hojas.

Dado que las hojas presentes en cada planta poseen diversos tamaños y, por ende, diferentes pesos, se sometió a pesaje distintos tamaños de hojas a fin de corroborar el peso de cada una y obtener el rendimiento promedio, en peso de materia verde de las hojas por planta de quínoa.

En promedio, cada planta de quínoa otorga $31,6 \mathrm{~g}$ de hojas de quínoa frescas, equivalente a $280 \mathrm{~kg} / \mathrm{ha}$ de hojas en fresco. Según la teoría de 
Cornejo (1976) y lo corroborado en la presente experiencia, las hojas rindieron cerca de un $15 \%$ de materia seca, por lo que los $280 \mathrm{~kg}$ de hojas frescas rendirían $42 \mathrm{~kg}$ de harina de hojas de quínoa. En base a lo anterior, si se realizase el proceso de cosecha y transformación 4 veces al mes, se obtendrían $168 \mathrm{~kg}$ de harina de hojas de quínoa por hectárea cultivada, según la variedad de quínoa y las condiciones ambientales. En los días siguientes a recolección de las hojas, las plantas se encontraban vigorosas, ya que se procuró no excederse en la extracción, para no afectar la producción de granos de quínoa ni su morfología. Es necesario evaluar el efecto que tendría esta práctica realizada con determinada frecuencia durante el crecimiento de las plantas. La información disponible en cuanto a la frecuencia, intensidad y técnica para la recolección de hojas tiernas es casi inexistente. Este know how se mantiene en las culturas alto andinas pero no ha sido sistematizada para buscar beneficios económicos de esta práctica. Esta podría significar una fuente de ingresos para los productores y aprovechamiento de proteínas de origen vegetal de buena calidad para consumo humano y animal.

\section{Valor nutricional de las hojas de quínoa deshidratadas}

El análisis químico proximal determinó que por cada 100 g de materia seca estas hojas de quínoa presentan 25,9 g de proteínas, 23,6 $\mathrm{g}$ de cenizas y $33,1 \mathrm{~g}$ de extractos no nitrogenados (Tabla 4). El contenido proteico en las muestras analizadas es mayor a lo establecido por Cornejo (1976) para otras variedades de quínoa ( 25,9 vs. $21 \%$ ). Esta diferencia se puede deber al origen (Perú y Bolivia) y momento de cosecha, ya que al ser cosechadas a los 60-70 días de su estado fenológico se encontraban en pleno crecimiento y estar formando masa vegetal, lo que implica que poseían gran cantidad de nitrógeno en su estructura.

Los porcentajes de inclusión de harina de hojas de quínoa utilizados en una porción de $50 \mathrm{~g}$ (porción de la mayoría de las galletas comerciales a base de avena), aportan más del 10\% de la DDR de proteínas requeridas por un adulto (50 g según el código alimentario) (Tabla 5).

La harina de quínoa deshidratada presenta un fuerte color verde y tiene un sabor amargo característico. Estas características son diferentes según la variedad de quínoa ya que la pigmentación

Tabla 4. Composición químico-proximal de hojas quínoa/100 gramos en seco.

\begin{tabular}{lcc}
\hline & 100 gramos & Referencia \\
\hline Humedad & 8,3 & PQM 001 revisión 06, 2014 basado en NCh 841, of 78 \\
Proteínas & 25,9 & PQM revisión 07, 2015 basado en PTR-711,02-173 ISP, 2009. \\
Lípidos & 4,8 & PQM 003 revisión 05, 2012 basado en procedimiento PTR-701.04-156 de ISP, 1998. \\
Cenizas & 23,6 & PQM 004 revisión 05, 2012 basado en NCh 842, of 78. \\
Fibra cruda & 4,3 & PQM 005 revisión 06, 2015 basado en AOAC, 962,09, 2010. \\
Extracto no nitrogenado & 33,1 & PQM 006 revisión 01, 2014 basado en Schimindt - Hebbel, página 32- 1981. \\
Calorías (kcal/ 100 g) & 279 & PQM 007 revisión 01, 2014 basado en Schmindt - Hebbel, página 31, 1981. \\
\hline Fuente: Departamento de Ciencia y Tecnología de los Alimentos, Universidad de Santiago de Chile, 2016.
\end{tabular}

Tabla 5. Contenido de proteína, según porcentajes de inclusión de harina de hojas de quínoa, para cada formulación.

\begin{tabular}{ccccccc}
\hline \multirow{2}{*}{$\%$ Inclusión } & \multicolumn{5}{c}{ Proteína presente en los ingredientes $(\mathrm{g})$} \\
\cline { 2 - 5 } & Quínoa & Avena & Harina trigo & Total & $\begin{array}{c}\text { Proteínas por porción } \\
(50 \mathrm{~g})\end{array}$ & DDR Proteínas \\
\hline 10 & 4,27 & 13,4 & 6,5 & 24,2 & 5,15 & $10,3 \% \uparrow$ \\
15 & 6,4 & 12,7 & 6,5 & 25,6 & 5,45 & $10,9 \% \uparrow$ \\
20 & 8,55 & 11,96 & 6,5 & 27 & 5,75 & $11,5 \% \uparrow$ \\
0 & - & 15 & 6,5 & 21,5 & 4,57 & $9,1 \% \downarrow$ \\
\hline
\end{tabular}


de la hoja puede ir desde verde oscuro a rojo (FAO, 2013).

\section{Evaluación sensorial}

Para todos los parámetros evaluados a través de las pruebas hedónicas (color, olor, textura, sabor y percepción general), a medida que el porcentaje de adición de harina de hojas de quínoa se aumentó en las formulaciones, mayor fue el rechazo. Sin embargo, este rechazo fue menor en aquellos tratamientos con hojas de quínoa lavadas (Tabla 6).

Color. La adición de harina de hojas de quínoa afecta de forma significativa y negativa el nivel de agrado, pues el tratamiento testigo presentó un color café claro y obtuvo gran aceptación; en cambio, los 6 tratamientos restantes presentaron diferentes tonos verdes que causaron gran rechazo en los panelistas. Esto se podría deber al fuerte pigmento verde presente en las hojas de quínoa deshidratadas.

$\mathrm{Al}$ analizar la desviación estándar de las medias, el tratamiento testigo presentó la menor dispersión de respuestas y la media más alta, lo cual hace de este tratamiento el más confiable y aceptable. De entre los tratamientos con harina de hoja de quínoa en su formulación, el tratamiento 7 fue el más aceptado y confiable.

Olor. La adición de harina de hojas de quínoa afectó de forma significativa el nivel de agrado de los panelistas, pues solo el tratamiento 7 fue percibido de forma similar al tratamiento control.

Respecto a las medidas de dispersión, el tratamiento 7 presentó la menor desviación estándar y mayor media, por lo cual es el que causó mayor agrado a los panelistas y, a la vez, el más confiable en lo que respecta a la variación de los valores de su media. En segundo lugar, se encuentra el tratamiento 6, el que a pesar de obtener la misma dispersión de respuestas que el tratamiento 7 , obtuvo una menor media de aceptación.

Textura. El testigo obtuvo un puntaje de indiferencia (no me gusta ni me disgusta) y la adición de harina de hojas de quínoa solo afecta de forma negativa y significativa en el nivel de agrado de la textura del tratamiento 1 .

Respecto a sus medidas de dispersión, el tratamiento 7 presentó la menor dispersión de respuestas $(\mathrm{DE}=0,98)$ y la media más alta $(5,00)$, siendo más confiables y aceptables. El segundo tratamiento más aceptado y confiable fue el tratamiento 6 (DE=1,31 y media 4,39).

Sabor. En este atributo se presenta la misma tendencia ocurrida en los atributos analizados anteriormente. El tratamiento testigo presentó diferencias significativas con los tratamientos 1,2 y 3 , siendo percibido de forma similar a los tratamientos 5,6 y 7 , es decir, aquellos porcentajes mejoran las propiedades nutricionales de los tratamientos sin afectar de forma significativa y negativa en su sabor. Esto podría atribuirse al fuerte pigmento verde presente en las hojas de quínoa deshidratadas, que produciría el amargor a las formulaciones, afectando de manera negativa en la aceptación del consumidor.

Finalmente, al analizar la desviación estándar de las medias totales, el tratamiento 7 presentó la menor dispersión de respuestas $(\mathrm{DE}=0,93)$ y la mayor media, por lo cual es posible afirmar que fue el tratamiento más aceptado y más confiable.

Percepción general de las galletas. Las medias de aceptación de los panelistas para cada

Tabla 6. Evaluación sensorial de los panelistas, para los diferentes tratamientos en cada variable evaluada.

\begin{tabular}{|c|c|c|c|c|c|c|c|c|c|c|}
\hline \multirow[b]{2}{*}{ Tratamiento } & \multicolumn{2}{|c|}{ Color } & \multicolumn{2}{|c|}{ Olor } & \multicolumn{2}{|c|}{ Textura } & \multicolumn{2}{|c|}{ Sabor } & \multicolumn{2}{|c|}{ Percepción general } \\
\hline & Media & $\mathrm{DE}$ & Media & $\mathrm{DE}$ & Media & $\mathrm{DE}$ & Media & $\mathrm{DE}$ & Media & $\mathrm{DE}$ \\
\hline $\mathrm{T} 1$ & $2,72^{1}$ & 1,35 & $3,91^{1}$ & 1,4 & 3,34 & 1,55 & 2,85 & 1,63 & 3,08 & 1,4 \\
\hline $\mathrm{T} 2$ & $2,91^{1}$ & 1,41 & $4,00^{1}$ & 1,23 & $3,83^{1}$ & 1,53 & $3,61^{1}$ & 1,74 & $3,76^{1}$ & 1,39 \\
\hline $\mathrm{T} 3$ & $3,47^{2}$ & 1,28 & $4,32^{2}$ & 1,25 & $4,22^{1}$ & 1,33 & $3,82^{1}$ & 1,54 & $4^{1}$ & 1,32 \\
\hline T5 & $3,75^{2}$ & 1,4 & $4,49^{2}$ & 1,19 & $4,46^{2}$ & 1,34 & $5^{2}$ & 1,09 & $4,86^{2}$ & 1,09 \\
\hline T6 & $3,79^{2}$ & 1,23 & $4,53^{2}$ & 1,11 & $4,39^{2}$ & 1,31 & $5,17^{2}$ & 1,06 & $5,01^{2}$ & 1,13 \\
\hline $\mathrm{T} 7$ & 4,35 & 1,22 & 4,82 & 1,11 & $5^{2}$ & 0,98 & $\mathbf{5 , 5 5 ^ { 2 }}$ & $\mathbf{0 , 9 3}$ & 5,75 & $\mathbf{0 , 8 2}$ \\
\hline T0 & 6,04 & $\mathbf{0 , 8 8}$ & 4,91 & 1,17 & $4,22^{1}$ & 1,29 & $5,17^{2}$ & 1,36 & $5,14^{2}$ & 1,22 \\
\hline
\end{tabular}

T1: 20\% hojas sin lavar, T2: 15\% hojas sin lavar; T3: 10\% hojas sin lavar; T4: 20\% hojas lavadas; T5: 15\% hojas lavadas; T6: 10\% hojas lavadas;

T0: Testigo. DE: desviación estándar.

Superíndices indican diferencias significativas por columna. 
tratamiento difieren entre sí. Los niveles de rechazo disminuyeron en aquellos tratamientos con menor porcentaje de adición de harina de hojas de quínoa $\mathrm{y}$, a la vez, este porcentaje de rechazo fue menor en aquellos tratamientos con las hojas de quínoa previamente lavadas.

Los resultados indican que la adición de harina de hojas de quínoa bajo el tratamiento "hojas lavadas" no afectó de forma negativa la percepción del agrado general que produjo a los panelistas para los tratamientos 5, 6 y 7 , si se compara con el nivel de agrado del tratamiento testigo. Por otro lado, la adición de la harina de hojas de quínoa bajo el tratamiento "hojas sin lavar" afecta de forma negativa y significativa en el nivel de agrado en los tratamientos 1,2 y 3.

En relación a las medidas de dispersión, el tratamiento 7 presentó la menor desviación estándar $(\mathrm{DE}=0,82)$ y la mayor media.

\section{Prueba de aceptación por ordenación}

Los tratamientos con inclusión de harina de hojas de quínoa con mayor puntuación y aceptación fueron los tratamientos 5,6 y 7 , correspondientes al tratamiento "hojas lavadas". Los tratamientos 1 , 2 y 3 presentaron la menor puntuación y, por ende, menor aceptación.

Los tratamientos 1,2,3 y 5 fueron significativamente inferiores en aceptación, respecto al control (tratamiento 0 ). El tratamiento 6, aunque fue percibido inferior al control, no lo fue de manera significativa. Lo mismo sucedió con el tratamiento 7, a pesar de que el panel consideró que el tratamiento 7 fue más aceptable respecto al control, esta diferencia no fue significativa.

\section{Aceptación y disposición de compra hacia otros productos con harina de hojas de quínoa}

Los datos obtenidos a través de la encuesta de aceptación, determinaron que un $81 \%$ del panel estaría dispuesto a adquirir productos enriquecidos o adicionados con hojas de quínoa deshidratadas. El 19\% restante declaró que no adquiriría dichos productos. En la sección comentarios del porqué el panelista no adquiriría productos con hojas de quínoa deshidratadas destacaron observaciones como: "Sabor amargo" y "No me agrada el color". Estos comentarios se deben, en gran medida, al hecho que ambos atributos fueron los más rechazados en la evaluación. En menor medida se encontraron comentarios como "No cuido mi alimentación" y "No consumo alimentos enriquecidos o adicionados".

Ante la pregunta: “ ¿Qué productos desearía enriquecer o adicionar con harina de hojas de quínoa?, del $81 \%$ del panel dispuesto a adquirir otros productos enriquecidos o adicionados con harina de hojas de quínoa, un 44\% afirmó que les gustaría adquirir pan con harina de hojas de quínoa, un $31 \%$ declaró que les gustaría adquirir barras de cereal y un $20 \%$ queques. Un $5 \%$ seleccionó "Otros", declarando que les gustaría adquirir salsas, arroz, pastas y té de hierbas con inclusión de harina de hojas de quínoa.

Los resultados de la encuesta aplicada determinaron que del $81 \%$ del panel dispuesto a adquirir otros productos enriquecidos o adicionados con harina de hojas de quínoa, un $83 \%$ del panel adquiriría harina de hojas de quínoa en formatos de 500 o $1.000 \mathrm{~g}$, para sus preparaciones panaderas caseras, si estos existiesen en supermercados u otros comercios y si tuvieran clara la forma de preparación y dosis. El 17\% restante declaró que no estaría dispuesto a adquirir dicho producto. Los comentarios más recurrentes ante esta respuesta fueron "No tengo tiempo para cocinar" y "No sabría cómo prepararlo".

\section{Conclusiones}

Es posible transformar las hojas tiernas de quínoa a harina de hojas de quínoa sin complejidad. También es posible incluirlas como ingrediente de buen valor nutricional a los alimentos en ciertos porcentajes. Deshidratar las hojas con luz solar no incurre en costos de procesamiento importantes, siendo una buena opción para los agricultores.

$\mathrm{El}$ rendimiento de materia verde a materia seca coincidió con la información establecida por la literatura, rindiendo un $15 \%$ aproximado de materia seca. La información obtenida del rendimiento de materia seca de las hojas tiernas se puede aplicar de manera sencilla a las distintas capacidades productivas de los agricultores.

El contenido proteico de las muestras fue superior al descrito por la literatura de Perú y Bolivia, para otras variedades; y es atribuido al origen y momento de cosecha.

En relación a la evaluación sensorial, se presentó la misma tendencia en las pruebas hedónicas por atributo y la prueba de aceptación por ordenación, en 
donde las galletas con mayor porcentaje de inclusión de harina de hojas de quínoa en sus formulaciones, presentaron mayor rechazo, en comparación a las galletas con menor porcentaje de inclusión y, a la vez, aquellas bajo el tratamiento "hojas sin lavar" fueron más rechazados que aquellos bajo el tratamiento "hojas lavadas".

El color y el sabor fueron los atributos que se vieron más afectados por la adición de la harina de hojas de quínoa a sus formulaciones. Esto se podría deber al fuerte pigmento verde presente en las hojas de quínoa deshidratadas y el amargor que otorgan a las formulaciones, afectando de manera negativa en la aceptación del consumidor. Sin embargo, al lavarlas eliminaron un poco de su pigmento y amargor, razón por la cual los tratamientos bajo el tratamiento "hojas de quínoa lavada" fueron más aceptados en todos sus atributos. Es necesario evaluar eventuales pérdidas de nutrientes en este lavado.

La prueba de aceptación por ordenación, sugieren que adicionar los alimentos al 15 y $10 \%$ bajo el tratamiento "hojas lavadas" mejora la calidad nutricional del producto, sin modificar de forma significativa su agrado hacia los panelistas. Agregar porcentajes mayores a la formulación u a otros productos podría afectar negativamente sus colores y sabores. Cabe destacar que la importancia de esta prueba radica en que nivel de agrado y preferencia por aceptación no es lo mismo, ya que un individuo puede compartir nivel de agrado respecto a diversos productos, pero a la hora de elegir, se inclinará por uno y no por todos.

Con la encuesta de aceptación fue posible determinar una buena disposición de compra por parte de los consumidores hacia otros productos adicionados o enriquecidos con harina de hojas quínoa, e incluso, existió disposición de compra hacia el producto en formato de 500 o 1.000 gramos si este existiese en supermercados con las indicaciones de uso apropiadas. Esto hace inferir que, con el marketing e información adecuada, productos adicionados/ enriquecidos con harina de hojas de quínoa tendrían buena aceptación en el mercado.

La opción de enriquecer/adicionar productos con harina de hojas de quínoa como una nueva alternativa alimentaria, en los porcentajes justos, que no afecten la calidad organoléptica de los alimentos, fue bastante aceptada, haciendo de los tratamientos con 15 y $10 \%$ de hojas lavadas, respectivamente, los preferidos por el consumidor. Sin embargo, en el presente estudio se utilizó una formulación base y no se intentó ocultar ningún efecto posible de la harina de hojas de quínoa hacia los tratamientos, por lo cual los resultados sugieren realizar estudios tendientes el método para disminuir y encubrir el amargor y color verde que otorga la harina de hojas de quínoa, de manera de poder aumentar los porcentajes de inclusión y aportar una mayor cantidad de proteínas a los alimentos.

La experimentación con diferentes variedades de quínoa con diferentes tonos de pigmentación en las hojas, con diferentes grados de frondosidad o genotipos con bajos niveles de saponina, puede permitir hacer de este recurso una fuente de ingresos para los productores. No obstante, es necesario identificar formas de cosecha que sean rentables para los agricultores, de modo que obtengan ganancias por sus granos de quínoa y por sus hojas, ya que la disponibilidad de materia prima para otorgarle un valor agregado está y actualmente es desaprovechada.

\section{Literatura Citada}

ANDINA - Agencia Peruana de Noticias.

2013. Hojas de quínoa también tienen alto valor nutritivo y se sirven en restaurantes. Lima, Perú. Disponible en: http:// www.andina.com.pe/agencia/noticia-hojas-quínoa-tambientienen-alto-valor-nutritivo-y-se-sirven-restaurantes-476318. aspx_Consultado: 20 diciembre de 2015.

Bazile, D.; Martinez, E.; Fuentes, F.; Chia, E.; Nambar, M.; Olguín, P.; Saa, C.; Thomet, M. y Vidal, A.

2014. La Quínoa en Chile. En: Bazile, D.; Bertero, D. y Nieto, C. (Eds.). Estado del Arte de la quínoa en el mundo en 2013. Santiago, Chile y Montpellier, Francia. pp. 477 - 503.
Carpenter, R.; Lyon, D. y Hasdell, T.

2003. Análisis Sensorial en el Desarrollo y Control de Alimentos. Segunda Edición. Editorial Acribia S.A. Zaragoza, España. 191 p.

Cornejo, G.

1976. Hojas de la quínoa (Chenopodium quinoa Willd.) fuente de proteína. En: Convención Internacional de Chenopodiaceas. 2da. Potosí, Bolivia. 26-29 abril. IICA. Serie informes de conferencias, cursos y reuniones. No. 96. Bolivia. pp. 177-180. 
Delatorre-Herrera, J.; Sánchez, M.; Delfino, I. y Oliva, M.I. 2013. La quínoa (Chenopodium quinoa Willd), un tesoro andino para el mundo. Idesia, 31 (2): 111-114.

FAO - Oficina Regional para América Latina y El Caribe. 2011. La quínoa: Cultivo milenario para contribuir a la seguridad alimentaria mundial. FAO. $66 \mathrm{p}$.

FAO - Oficina Regional para América Latina y El Caribe. 2013. Quínoa la planta. Vitacura, Chile. Disponible en: http://www. fao.org/quinoa-2013/what-is-quinoa/es/Consultado: 29 dic. 2015.

Fernández, C.

2005. Análisis sensorial de los alimentos. Disponible en: http://dcfernandezmudc.tripod.com/ Consultado: 10 enero de 2016.

Hernández, E.

2005. Evaluación Sensorial. Universidad Nacional y Abierta a Distancia - UNAD. Facultad de Ciencias Básicas e Ingeniería. Bogotá, Colombia. 125 p.

Meyhuay, M.

2013. Quínoa: Operaciones de Poscosecha. En: Mejia D. (Ed.). Instituto de Desarrollo Agroindustrial (INDDA). La Molina, Perú. 36 p.

Mujica, A.; Izquierdo, J.; Pierre, J.; Morón, C. y Jacobsen, S.-E. 1999. Reunión técnica y taller de formulación de producto regional sobre producción y nutrición humana en base a cultivos andinos. Arequipa, Perú. 182 p.
INE - Instituto Nacional de Estadísticas.

2016. Superficie Sembrada Año Agrícola 2011-2012.

Disponible en: www.ine.cl/canales/chile_estadistico/.../2012/ superficie_sembrada_2011_2012.pdf Consultado: 28 diciembre de 2015.

Pedrero, D. y Pangborn, R.

1989. Evaluación sensorial de los alimentos. Métodos analíticos. Primera edición. Distrito Federal, México. Editorial Alhambra Mexicana. 251 p.

Ramírez-Navas, $\mathrm{S}$.

2012. Análisis sensorial. Pruebas orientadas al consumidor. Revista RECITEIA, 12 (1): 83-102.

SERNAC - Servicio Nacional del Consumidor.

2003. Análisis de características nutricionales y rotulación de cereales en barra. Gobierno de Chile. Departamento de estudios. Santiago, Chile. 60 p.

Tonconi, J.

2015. Producción agrícola alimentaria y cambio climático: un análisis económico en el departamento de Puno, Perú. Idesia, 33 (2): 119-136.

Watts, B.; Ylimaki, G.; Jeffery, E. y Elías, L.

1992. Métodos sensoriales básicos para la evaluación de alimentos. Departamento de alimentos y nutrición. Facultad de Ecología Humana. Universidad de Manitoba, Winnipeg, Primera edición. Manitoba, Canadá. 172 p.

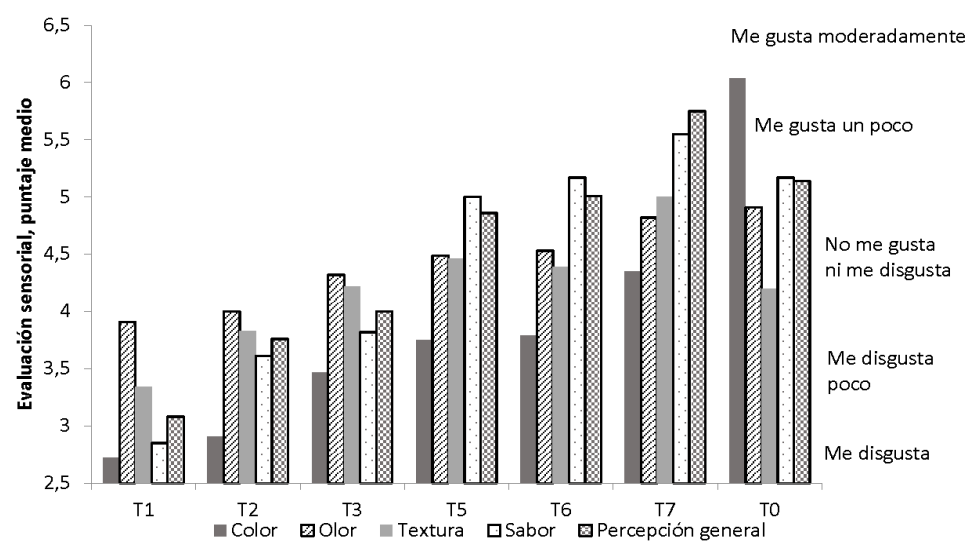

Figura 1. Puntajesmedios de evaluación sensorial, asignados por los panelistas a los diferentes tratamientos. T1: $20 \%$ hojas sin lavar, T2: 15\% hojas sin lavar; T3: 10\% hojas sin lavar; T4: 20\% hojas lavadas; T5: 15\% hojas lavadas; T6: 10\% hojas lavadas; T0: Testigo. 


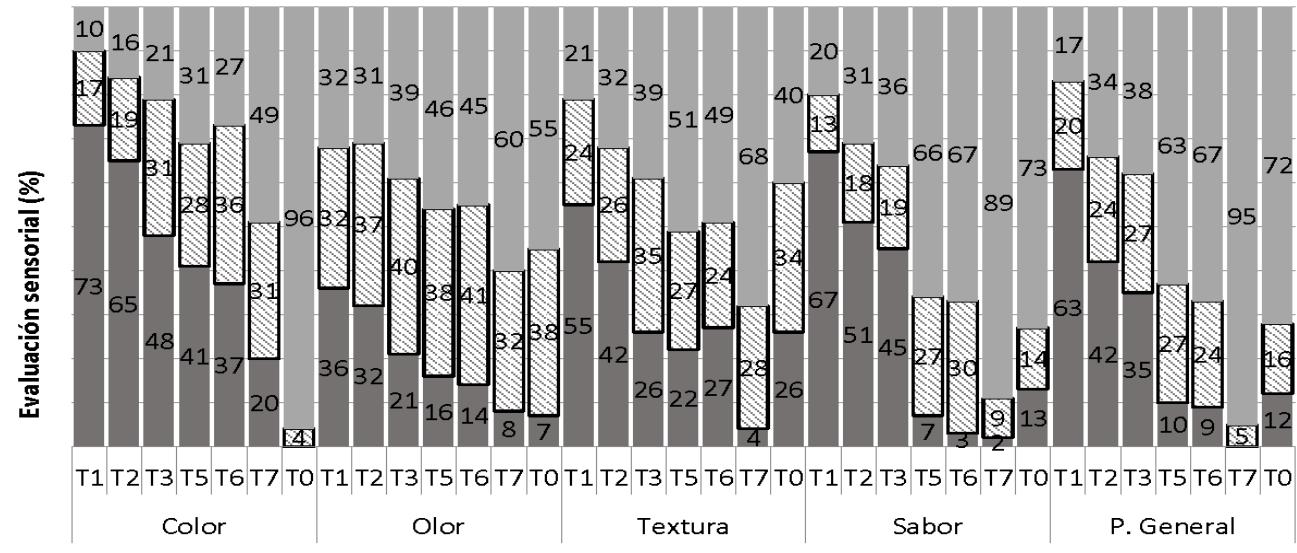

$\square$ Positivo $\mathbf{8}$ Neutro $\square$ Rechazo

Figura 2. Porcentajes de evaluación positiva, neutra y negativa del panel, para los diferentes tratamientos evaluados. T1: 20\% hojas sin lavar, T2: 15\% hojas sin lavar; T3: 10\% hojas sin lavar; T4: 20\% hojas lavadas; T5: 15\% hojas lavadas; T6: 10\% hojas lavadas; T0: Testigo. 